\title{
MYB and MYBL1 in adenoid cystic carcinoma: diversity in the mode of genomic rearrangement and transcripts
}

\author{
Yuki Togashi ${ }^{1,2,3} \cdot$ Akito Dobashi $^{1} \cdot$ Seiji Sakata ${ }^{1} \cdot$ Yukiko Sato $\circledast^{2}$ - Satoko Baba ${ }^{1} \cdot$ Akira Seto $^{4} \cdot$ Hiroki Mitani $^{4}$. \\ Kazuyoshi Kawabata ${ }^{4} \cdot$ Kengo Takeuchi $\mathbb{1}^{1,2,3}$
}

Received: 26 September 2017 / Revised: 28 November 2017 / Accepted: 3 December 2017 / Published online: 6 February 2018

(c) United States \& Canadian Academy of Pathology 2018

\begin{abstract}
MYB-NFIB and MYBLI-NFIB have been reported in $\sim 60 \%$ of adenoid cystic carcinoma cases, but driver alterations in the remaining $\sim 40 \%$ of adenoid cystic carcinoma remain unclear. We examined 100 adenoid cystic carcinoma cases for $M Y B$ and MYBL1 locus rearrangements by fluorescence in situ hybridization (FISH) with originally designed probe sets using formalin-fixed paraffin-embedded materials. Approximately one-third of samples were also analyzed by fusion transcriptspecific RT-PCR and capture RNA sequencing. In the 27 cases with frozen materials, MYB-NFIB and MYBL1-NFIB fusion transcripts were detected in $9(33 \%)$ and 6 cases $(22 \%)$ by RT-PCR, respectively. Meanwhile, high expression of MYB (18 cases, 67\%) or MYBL1 (9 cases, 33\%) was detected in all 27 cases in a mutually exclusive manner, regardless of its form (full-length, truncation, or fusion transcript). Interestingly, genomic rearrangements around the corresponding highlyexpressed gene were observed in all 27 cases by FISH, suggesting a causative relationship between genomic rearrangements and gene expression. Among the 100 cases, including additional 73 cases, 97 harbored genomic rearrangements in the MYB (73 cases) or MYBL1 locus (24 cases) including 10 cases with atypical FISH patterns undetectable through ordinary split FISH approaches: breakpoints far distant from MYB (5 cases) and a small NFIB locus insertion into the MYB (3 cases) or $M Y B L 1$ locus ( 2 cases). In clinicopathological analyses, histological grade, primary tumor size, and lymph node metastasis were identified as prognostic factors, whereas $M Y B / M Y B L 1$ rearrangements were not, but were associated with histological grade. In the present study, MYB or MYBL1 locus rearrangement was detected in nearly all adenoid cystic carcinoma cases, and therefore it would be a good diagnostic marker for adenoid cystic carcinoma. However, fusion transcript-specific RTPCR for MYB-NFIB and MYBL1-NFIB and ordinary split FISH assays for MYB and MYBL1 were less sensitive, and thus detection methods should be judiciously designed because of the diversity of rearrangement modes in adenoid cystic carcinoma.
\end{abstract}

Electronic supplementary material The online version of this article (https://doi.org/10.1038/s41379-018-0008-8) contains supplementary material, which is available to authorized users.

Kengo Takeuchi

kentakeuchi-tky@umin.net

1 Pathology Project for Molecular Targets, The Cancer Institute, Japanese Foundation for Cancer Research, 3-8-31 Ariake, Koto, Tokyo 135-8550, Japan

2 Division of Pathology, The Cancer Institute, Japanese Foundation for Cancer Research, 3-8-31 Ariake, Koto, Tokyo 135-8550, Japan

3 Department of JFCR Cancer Biology, Graduate School of Medical and Dental Sciences, Tokyo Medical and Dental University, 1-545 Yushima, Bunkyo, Tokyo 113-8510, Japan

4 Department of Head and Neck Oncology, The Cancer Institute Hospital, Japanese Foundation for Cancer Research, 3-8-31 Ariake, Koto, Tokyo 135-8550, Japan

\section{Introduction}

Adenoid cystic carcinoma is a rare salivary gland-type tumor that arises in exocrine glands including the salivary, lacrimal, mammary, and bronchial glands [1]. The median age at diagnosis is $\mathbf{5 4 . 6}$ years and the female ratio is $\sim 60 \%$ [1]. Although locoregional recurrence and distant metastasis are common, adenoid cystic carcinoma is an indolent cancer (5-year overall survival: 77\%, 10-year: 60\%, and 15-year: $45 \%)$ [1].

Histopathologically, the most characteristic architecture of adenoid cystic carcinoma is the cribriform pattern showing tumor nests with gland-like structures filled with a basophilic substance. The tubular pattern is comprised of glands containing inner epithelial and outer myoepithelial layers. In the solid pattern, tumor nests consist of 
monotonous proliferation of epithelial or myoepithelial tumor cells with occasional gland and gland-like structure formation. Depending on the case, these patterns are observed in a single tumor in different ratios. Other salivary-gland type tumors including basal cell adenoma, basal cell adenocarcinoma, pleomorphic adenoma, polymorphous low-grade adenocarcinoma, and epithelial-myoepithelial carcinoma may mimic these patterns of adenoid cystic carcinoma. Therefore, definitively diagnosing adenoid cystic carcinoma may be difficult, particularly when using small biopsy specimens.

In several salivary gland-type tumors, histopathological subtype-specific fusion genes have been identified, including PLAG1 and HMGA2 fusions in pleomorphic adenoma $[2,3], C R T C 1-M A M L 2$ in mucoepidermoid carcinoma [4, 5], ETV6-NTRK3 in secretory carcinoma [6], and EWSR1$A T F 1$ in clear cell carcinoma [7]. In adenoid cystic carcinoma, MYB-NFIB [8-11] and MYBLI-NFIB [12-14] were detected in $\sim 60 \%$ of cases [15]. However, driver alterations in the remaining $40 \%$ of adenoid cystic carcinoma remain unclear. In the present study, we aimed to clarify the significance of MYB family genes in the pathogenesis of adenoid cystic carcinoma using histopathology, molecular biology, and bioinformatics for 100 adenoid cystic carcinoma cases.

\section{Materials and methods}

\section{Case selection and histological review}

Three pathologists histopathologically reviewed the slides of 123 patients with adenoid cystic carcinoma diagnosed and treated in the Cancer Institute Hospital (Tokyo, Japan) between January 2005 and October 2016. Twenty-three cases were excluded because a consensus diagnosis of adenoid cystic carcinoma was not obtained in 14 cases and archival samples were insufficient in 9 cases (Supplementary Table 1). This study was approved by the Institutional Review Board of Japanese Foundation for Cancer Research.

The three pathologists independently graded the remaining 100 cases based on the proportion of solid component; grade 1 was assigned when the solid component was $<30 \%$ of the tumor area; grade $2,30 \% \leq$ solid component $<50 \%$; and grade $3,50 \% \leq$ solid component. When an area of histological transformation was identified, the suffix $\mathrm{t}$ was added to the grade. Each pathologist's grade was defined as the consensus grade when it was agreed upon by 2 or 3 pathologists. Graded cases were grouped into low-grade (grades 1 and 2) and high-grade cases (grades 3, 1t, 2t, and 3t) for statistical analyses.
Fluorescence in situ hybridization (FISH)

Unstained formalin-fixed paraffin-embedded sections (4$\mu \mathrm{m}$-thick) were subjected to hybridization with bacterial artificial chromosome clone-derived probes for $M Y B$, $M Y B L 1$, and NFIB. The clones used are shown in Supplementary Table 2 and genomic regions covered by the probes are illustrated in Supplementary Figure 1. For $M Y B$, we developed two probe sets. One was designated as the widerange $M Y B$ probe set covering $11.5 \mathrm{Mb}$ upstream to $8.4 \mathrm{Mb}$ downstream of $M Y B$ (Supplementary Figure 1A). The other was a conventionally designed probe set covering $386.7 \mathrm{~kb}$ upstream to $136.6 \mathrm{~kb}$ downstream of $M Y B$, the region similar to those covered by previously reported and commercially available $M Y B$ probe sets (Supplementary Figure 1B) $[11,16-20]$. The conventionally designed probe set was used for initial screening. Cases negative in both $M Y B$ and MYBL1 split FISH assays were again examined for $M Y B$ locus rearrangement using the wide-range probe set. Probe sets for MYBL1 and NFIB were designed to cover $681.1 \mathrm{~kb}$ and $631.8 \mathrm{~kb}$, respectively (Supplementary Figure 1C, D). Hybridized slides were stained with DAPI and examined using a BX51 fluorescence microscope (Olympus, Tokyo, Japan).

\section{Immunohistochemistry}

MYB protein was detected on unstained 4- $\mu$ m-thick formalin-fixed paraffin-embedded sections after deparaffinization with xylene using a rabbit monoclonal antibody to c-Myb (EP769Y) (Abcam, Cambridge, UK) at a dilution of $1: 200$.

\section{Total RNA extraction and CDNA synthesis}

For fresh frozen specimens, total RNA was extracted with RNeasy Mini Kit (QIAGEN, Hilden, Germany) and used to synthesize cDNA with SuperScript III Reverse Transcriptase (Thermo Fisher Scientific, Waltham, MA, USA) and random primers. For formalin-fixed paraffin-embedded specimens, regions of interest (tumor and normal regions) on $10-\mu \mathrm{m}$-thick sliced histopathological sections were manually dissected using disposable needles under a stereomicroscope and processed with RecoverAll Total Nucleic Acid Isolation (Thermo Fisher Scientific) to extract total RNA.

\section{Multiplex RT-PCR for MYB-NFIB and MYBL1-NFIB fusions}

To amplify MYB-NFIB and MYBL1-NFIB fusion cDNAs, multiplex reverse-transcription polymerase chain reaction (RT-PCR) analysis was conducted using AmpliTaq Gold 
Table 1 Patient characteristics

\begin{tabular}{|c|c|c|c|c|c|c|}
\hline & \multicolumn{3}{|c|}{ Head and neck (including orbital region) $(N=81)$} & \multicolumn{3}{|c|}{ All $(N=100)$} \\
\hline & Total & $\begin{array}{l}\text { MYB-type } \\
(N=64)\end{array}$ & $\begin{array}{l}\text { MYBL1-type } \\
(N=17)\end{array}$ & Total & $\begin{array}{l}\text { MYB-type } \\
(N=76)\end{array}$ & $\begin{array}{l}\text { MYBL1-type } \\
(N=24)\end{array}$ \\
\hline Female/male & $54 / 27$ & $43 / 21$ & $11 / 6$ & $71 / 29$ & $55 / 21$ & $16 / 8$ \\
\hline Age, median (range) & $\begin{array}{l}54 \\
(15-80)\end{array}$ & $53(15-78)$ & $59(34-80)$ & $\begin{array}{l}57 \\
(15-85)\end{array}$ & $54(15-85)$ & $60(34-80)$ \\
\hline $\begin{array}{l}\text { Primary tumor size } \\
(\mathrm{mm}), \text { median } \\
\text { (range) }(N=74)\end{array}$ & $31(8-79)$ & $32(8-79)$ & $30(13-50)$ & NA & NA & NA \\
\hline $\begin{array}{l}\text { Primary distant } \\
\text { metastasis }\end{array}$ & $9 / 81$ & $7 / 64$ & $2 / 17$ & $9 / 98$ & $7 / 74$ & $2 / 24$ \\
\hline $\begin{array}{l}\text { Primary lymph node } \\
\text { metastasis }\end{array}$ & $5 / 81$ & $5 / 64$ & $0 / 17$ & $7 / 99$ & $6 / 75$ & $1 / 24$ \\
\hline \multicolumn{7}{|c|}{ Stage (UICC 8th) $(\%)(N=69)$} \\
\hline I & $6(9)$ & $4(7)$ & $2(13)$ & NA & NA & NA \\
\hline II & $12(17)$ & $8(15)$ & $4(27)$ & NA & NA & NA \\
\hline III & $28(41)$ & $22(41)$ & $6(40)$ & NA & NA & NA \\
\hline IVA/B/C & $\begin{array}{l}12 / 3 / 8 \\
(33)\end{array}$ & $11 / 3 / 6(37)$ & $1 / 0 / 2(20)$ & NA & NA & NA \\
\hline \multicolumn{7}{|l|}{ Histological grade } \\
\hline Low $(1 / 2)$ & $63(53 / 10)$ & $47(38 / 9)$ & $16(15 / 1)$ & $78(65 / 13)$ & $57(45 / 12)$ & $21(20 / 1)$ \\
\hline High $(3 / 1 t / 2 t / 3 t)$ & $\begin{array}{l}18(12 / 4 / \\
1 / 1)\end{array}$ & $\begin{array}{l}17(11 / 4 / 1 / \\
1)\end{array}$ & $1(1 / 0 / 0 / 0)$ & $\begin{array}{l}22(15 / 5 / \\
1 / 1)\end{array}$ & $\begin{array}{l}19(12 / 5 / 1 / \\
1)\end{array}$ & $3(3 / 0 / 0 / 0)$ \\
\hline
\end{tabular}

NA not applicable

DNA polymerase (Thermo Fisher Scientific) and the primers shown in Supplementary Table 3. The template cDNAs were incubated at $94{ }^{\circ} \mathrm{C}$ for $10 \mathrm{~min}$ and then subjected to 35 cycles of denaturation at $94{ }^{\circ} \mathrm{C}$ for $1 \mathrm{~min}$, annealing at $64{ }^{\circ} \mathrm{C}$ for $1 \mathrm{~min}$, and polymerization at $72{ }^{\circ} \mathrm{C}$ for $1 \mathrm{~min}$. The PCR products were separated by agarose gel electrophoresis, followed by direct sequencing or subcloning, if needed.

\section{3'-Rapid amplification of CDNA ends for MYB and MYBL1}

For cases negative in the multiplex RT-PCR for $M Y B-N F I B$ and $M Y B L 1-N F I B$ fusions, 3'-rapid amplification of cDNA ends was performed with one of the two forward primers designed for $M Y B$ and MYBL1 (Supplementary Table 3) under previously reported conditions [21]. Amplified products were subjected to direct sequencing.

\section{Capture RNA sequencing}

TruSeq RNA Access Library Prep Kit (Illumina, San Diego, CA, USA), which includes biotinylated capture probes targeting coding RNA, was used for coding RNA sequencing with an input of $20 \mathrm{ng}$ total RNA from fresh frozen specimens. TruSight RNA Pan-Cancer Panel (Illumina) was used for RNA sequencing targeted to 1385 cancer-related genes with an input of $100 \mathrm{ng}$ total RNA from formalinfixed paraffin-embedded specimens. All samples were prepared according to the manufacturer's instructions.

\section{RT-PCR for MPZL1-NFIB, MPDZ-MYB, TRAM1-MYBL1, MYBL1-RAD51B, and RAD51B-AHI1}

To confirm the fusion transcripts detected by coding RNA sequencing, RT-PCR was performed using Platinum Taq DNA Polymerase High Fidelity (Thermo Fisher Scientific) and the specific primer sets shown in Supplementary Table 3.

\section{Sequencing analyses of rearrangements of $M Y B$ with downstream regions of NFIB}

For the three cases in which fusion transcripts of $M Y B$ exon 14 to downstream regions of $N F I B$ were detected by capture RNA sequencing (ACC005, ACC008, and ACC089), genomic PCR was performed with the primers shown in Supplementary Table 3 using LA Taq (TaKaRa Bio, Shiga, Japan). The PCR mixture was incubated at $94{ }^{\circ} \mathrm{C}$ for $1 \mathrm{~min}$ and then subjected to 30 cycles of denaturation at $98{ }^{\circ} \mathrm{C}$ for $10 \mathrm{~s}$, annealing, and polymerization at $68^{\circ} \mathrm{C}$ for $5 \mathrm{~min}$. The PCR products were separated by agarose gel electrophoresis, followed by direct sequencing. 


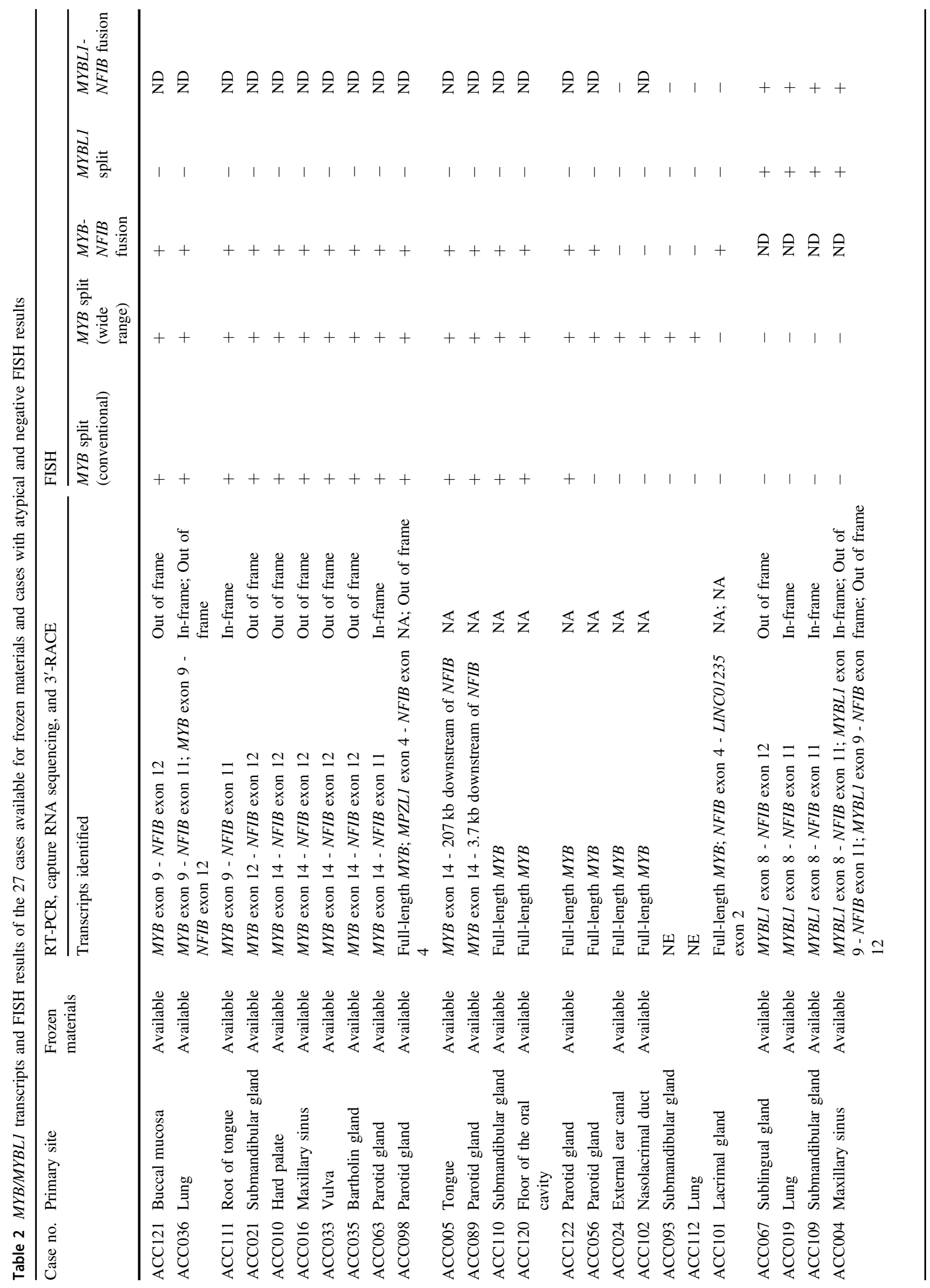




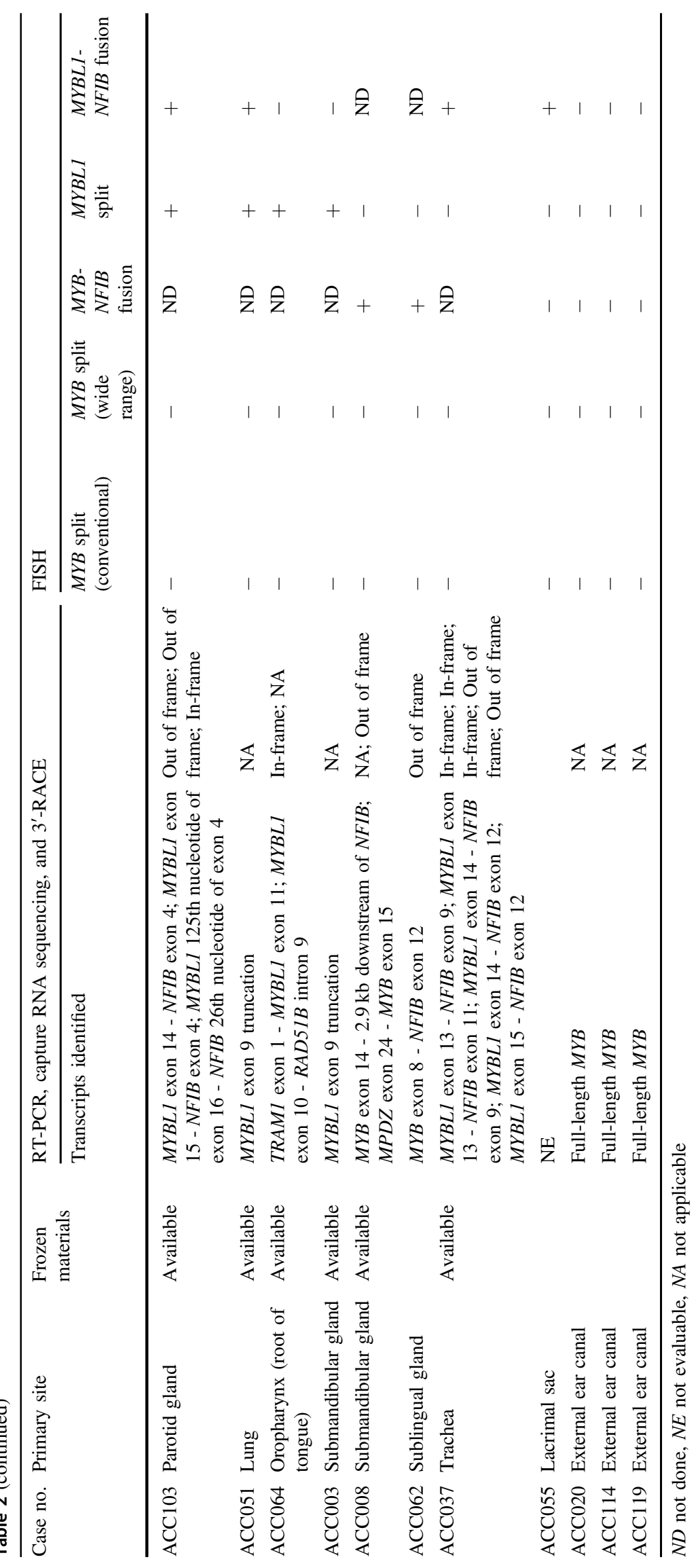




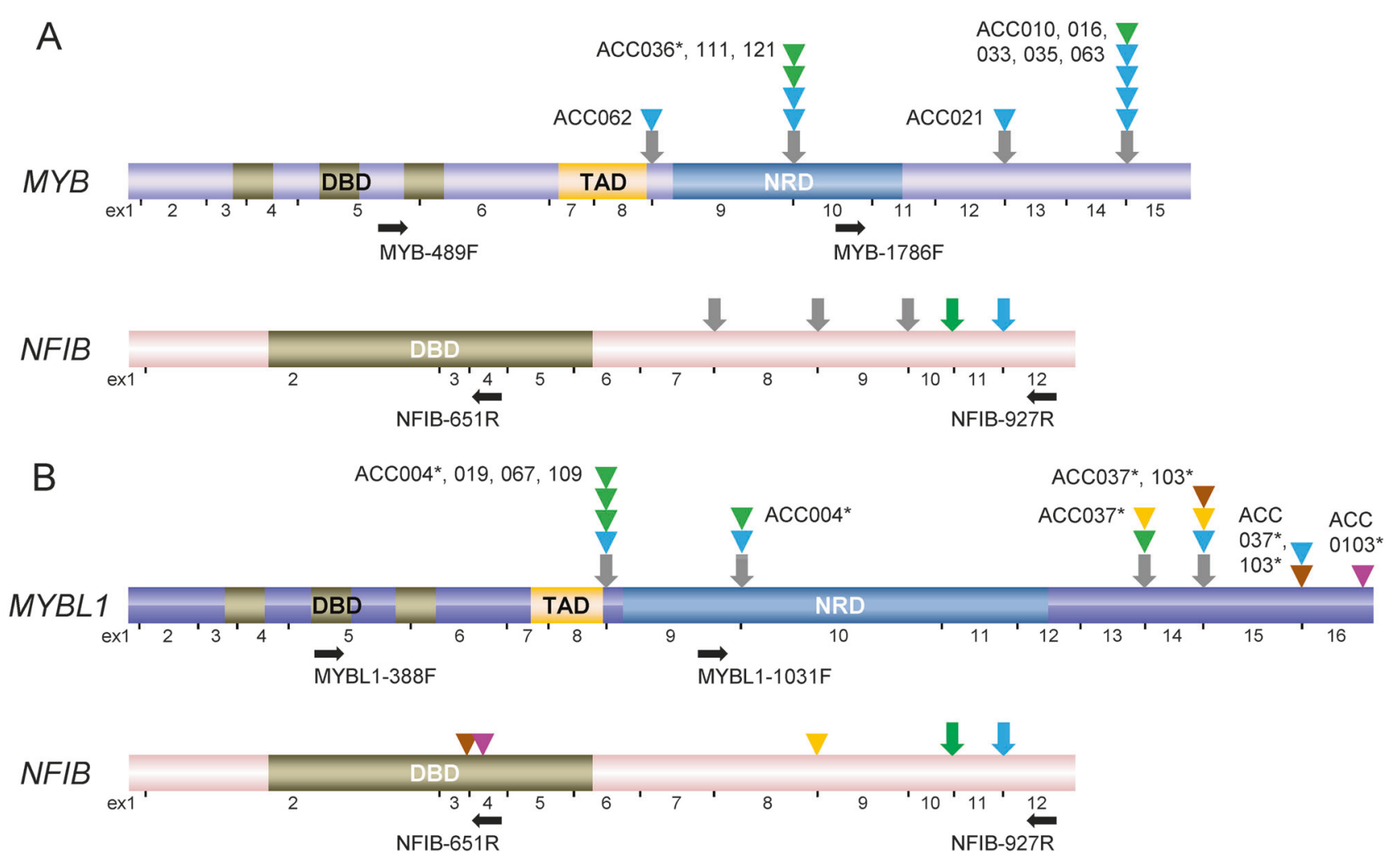

Fig. 1 Breakpoints in $M Y B-N F I B$ and MYBL1-NFIB fusion transcripts. Breakpoints in $M Y B$ and $N F I B$ (a) and MYBLI and NFIB (b) found to be fused to each other are shown in the same color. Arrows and triangles represent the breakpoints reported and those detected in the present study, respectively. Cases with asterisks showed alternative

splicing. The primer locations for multiplex RT-PCR are also shown. Note that all fusion-positive cases retained $M Y B$ or $M Y B L 1$ DNA binding domain (DBD) and transactivation domain (TAD). NRD negative regulatory domain

\section{Sequence data analysis}

Quality control was performed on fastq files using FASTXToolkit version 0.0 .13 (http://hannonlab.cshl.edu/fastx toolkit/index.html), PRINSEQ version 0.20.4 [22], and cmpfastq_pe (http://compbio.brc.iop.kcl.ac.uk/software/ cmpfastq_pe.php) software. Sequenced reads containing $<80 \%$ of bases with a quality score of Q20 or higher were deleted. Bases at read ends with $\mathrm{N}$ sequences or whose quality scores were less than Q20 were removed. Among the remaining reads, those shorter than 20 base pairs (bp) and the resulting non-paired reads were deleted. The qualified reads were mapped to hg 19 with STAR version 2.4.2a [23]. Fusion gene analyses were performed with FusionCatcher [24] and in-house programs. Expression analyses for $M Y B$ and $M Y B L 1$ were performed using R version 3.3.2 [25] and HTSeq version 0.6.1 [26].

\section{Statistics analysis}

Fisher's exact test (two-tailed) was used to analyze the differences between $M Y B$ and MYBL1 locus rearrangementpositive head and neck adenoid cystic carcinoma $(N=78)$. For histological grade, Mann-Whitney $U$ test was also used.

To assess the statistical significance of variables affecting overall survival, the log-rank test was employed for univariate analysis. Multivariate analysis was performed on prognostic factors with significant difference in univariate analysis $(P$ value $<0.05)$, using the Cox proportional hazard model. Univariate analysis was carried out with GraphPad Prism ${ }^{\circledR}$ Version 6.05 (GraphPad, Inc., La Jolla, CA, USA) and multivariate analysis was performed using $\mathrm{R}$ version 3.4.1 and survival version 2.38 .

\section{Results}

\section{Histopathological grading}

In the 100 cases with adenoid cystic carcinoma, there were 71 female and 29 male patients. The median age of onset was 56.8 years (range: 14.9-84.7). The sites of origin included major salivary glands $(N=40)$, minor salivary glands $(N=37)$, and other sites $(N=23)$. The number of cases of head and neck (including four cases of orbital region) were 81 (Table 1; Supplementary Table 4). The 100 cases were histopathologically classified as follows: 65 cases as grade 1,13 as grade 2,15 as grade 3 , five as grade 

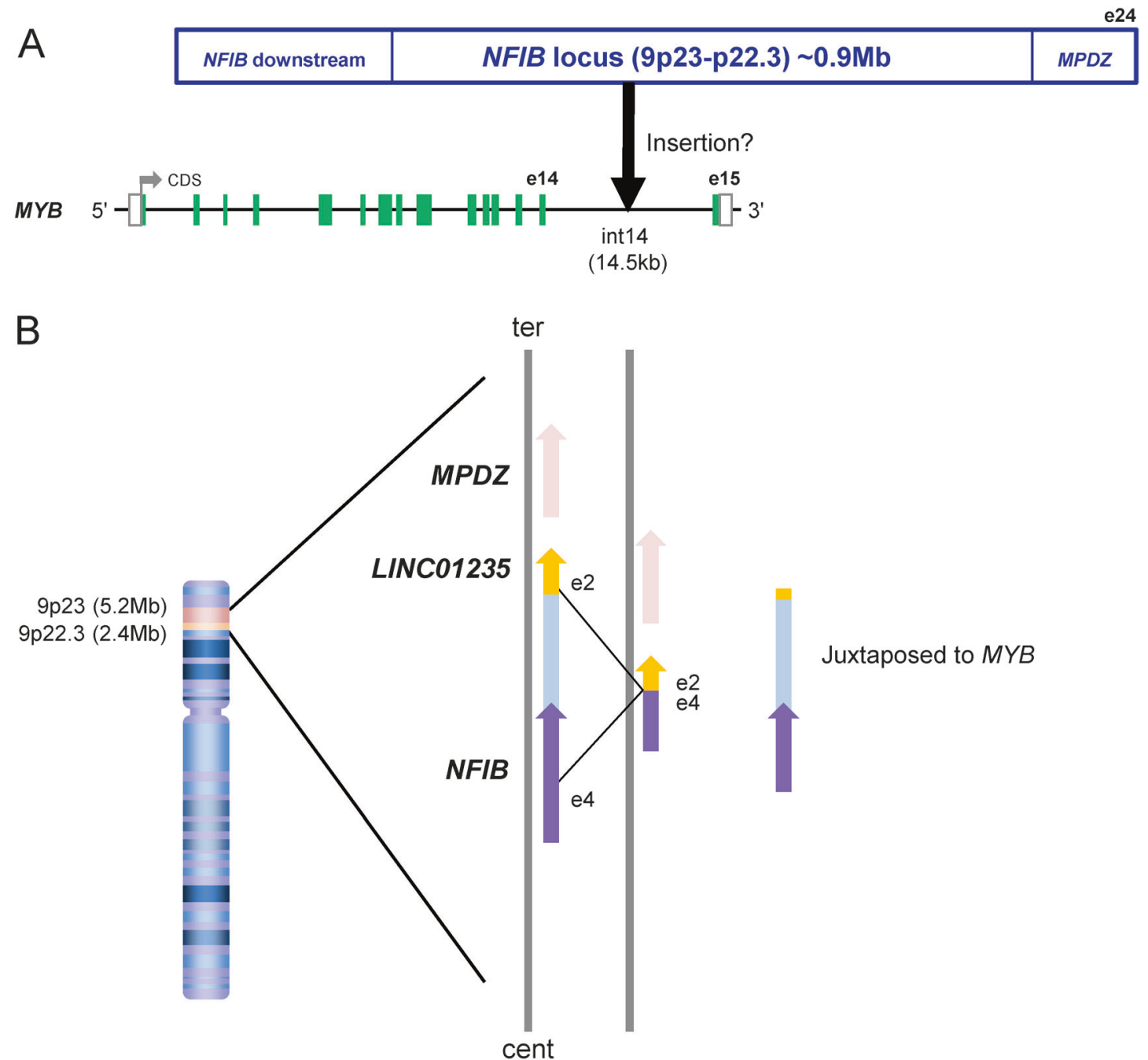

Juxtaposed to $M Y B$

Fig. 2 Estimated genomic rearrangements in $M Y B$ split FISH-negative but $M Y B-N F I B$ fusion FISH-positive cases. a Transcripts of $M Y B$ exon $14-N F I B \quad 3^{\prime}$ downstream and $M P D Z$ exon $24-M Y B$ exon 15 were detected in ACC008, suggesting an $\sim 0.9 \mathrm{Mb}$ insertion of the NFIB locus into MYB intron 14. b In ACC101, a transcript of NFIB exon 4-

$1 \mathrm{t}$, one as grade $2 \mathrm{t}$, and one as grade $3 \mathrm{t}$, resulting in 78 lowand 22 high-grade cases. In 81 head-and-neck cases, 63 and 18 cases were graded as low and high, respectively (Table 1).

\section{Expression of MYB and MYBL1 transcripts in the 27 cases with available frozen materials}

We developed a multiplex RT-PCR system that could theoretically detect all possible fusion variants coding for the $M Y B-N F I B$ or MYBL1-NFIB fusion protein. Among the 27 cases for which frozen materials were available, $9(33 \%)$ and $6(22 \%)$ cases were found to express MYB-NFIB and MYBL1-NFIB fusion transcripts, respectively. In the remaining 12 (44\%) cases, neither transcript was expressed (Table 2, Fig. 1, and Supplementary Figure 2).
$L I N C 01235$ exon 2 was identified. Therefore, a part of the NFIB locus, which consisted of the $N F I B$ sequence from intron 4 , intergenic region (gray) between NFIB and LINCO1235, and LINC01235 intron 1, was likely to juxtapose the $M Y B$ locus. Not to scale

Next, we performed a conventional 3'-rapid amplification of cDNA ends assay and coding RNA sequencing using next-generation sequencing. Interestingly, all 27 cases were found to highly express either MYB (18 cases, 67\%) or MYBL1 (9 cases, $33 \%$ ) in a mutually exclusive manner, and three additional MYB (ACC005, ACC008, and ACC089) and one additional MYBL1 (ACC064) fusion transcripts were identified in the 12 multiplex RT-PCR-negative cases (Table 2). In the three additionally identified $M Y B$ transcripts, $M Y B$ exon 14 fused to $9 \mathrm{p} 23$ intergenic sequences, $207 \mathrm{~kb}$ (ACC005), $2.9 \mathrm{~kb}$ (ACC008), and $3.8 \mathrm{~kb}$ (ACC089) downstream of the $3^{\prime}$ untranslated region (UTR) end of NFIB (Supplementary Figure 3). The genomic breakpoints in $M Y B$ intron 14 were at $4.4 \mathrm{~kb}$ (ACC005), $0.7 \mathrm{~kb}$ (ACC008), and $4.2 \mathrm{~kb}$ (ACC089) downstream from the end of MYB exon 14 (Supplementary Figure 3). In ACC008, an 


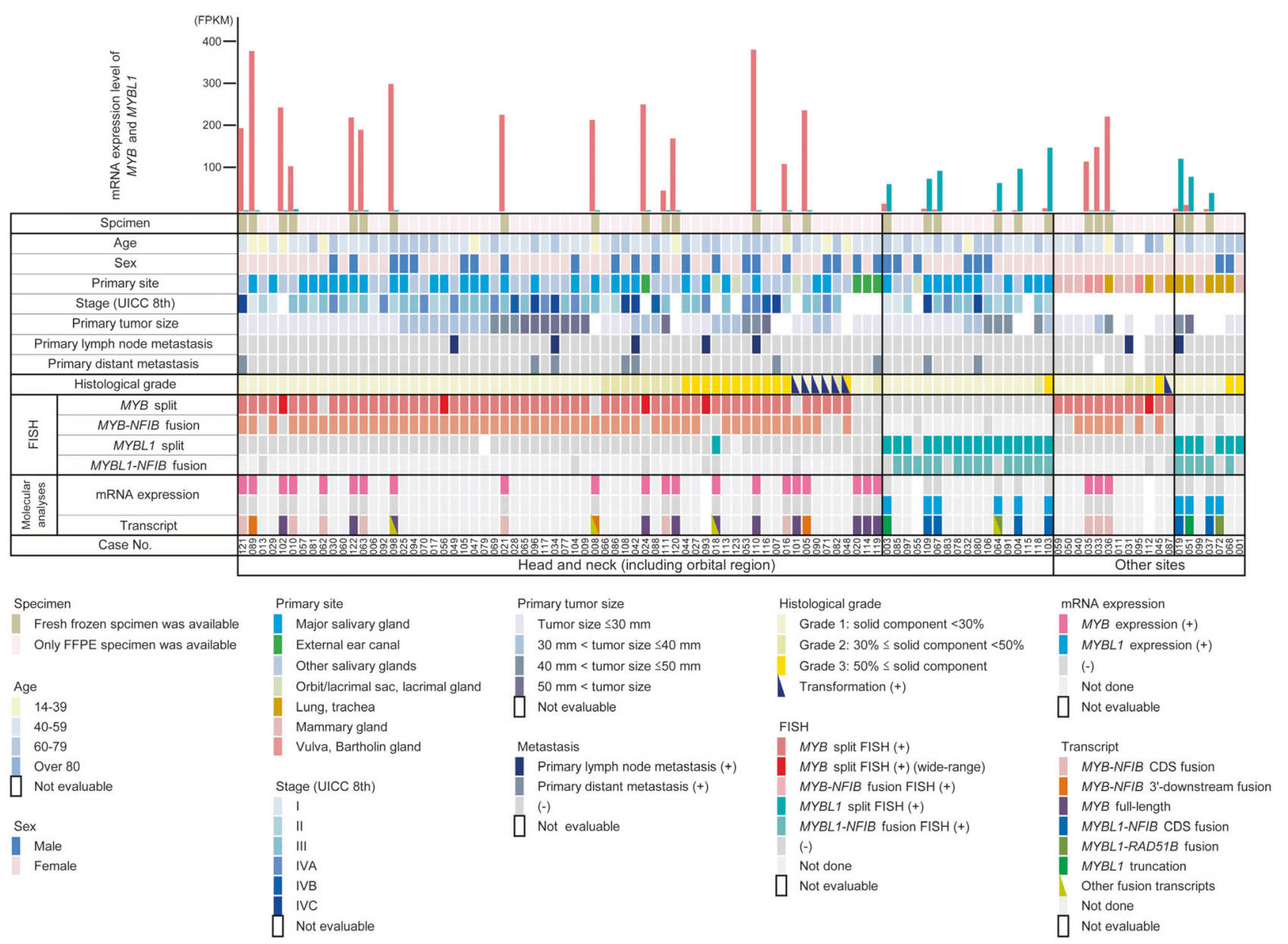

Fig. 3 Clinicopathological and genetic landscape of 100 cases with adenoid cystic carcinoma. At the top of the figure, $M Y B$ or $M Y B L 1$ expression level is shown using fragments per kilobase of exon per million mapped fragments (FPKM) (pink bar: MYB, green bar: MYBL1)

$\sim 0.9 \mathrm{Mb}$ fragment of chromosome 9 was considered inserted in $M Y B$ intron 14 because the following two fusion transcripts were identified: $M Y B$ exon 14 fused to $3.6 \mathrm{~kb}$ downstream of the NFIB $3^{\prime} \mathrm{UTR}$ and MPDZ exon 24 fused to $M Y B$ exon 15 (Fig. 2a; Supplementary Figure 4A). In ACC064, MYBL1 exon 10-RAD51B intron 9 and TRAM1 exon 1-MYBL1 exon 11 fusion transcripts were expressed and confirmed by specific RT-PCR (Supplementary Figure 4B). MYBL1 and RAD51B were fused to each other in the opposite direction in this case (Supplementary Figure 5). The genomic breakpoint of MYBL1 was in intron $10,87 \mathrm{bp}$ downstream of exon 10, which was fused to intron 9 of $R A D 51 B, 49.5 \mathrm{~kb}$ downstream of exon 9 (Supplementary Figure 5). As MYBL1, TRAM1, and RAD51B are located at $8 \mathrm{q} 13.1,8 \mathrm{q} 13.3$, and $14 \mathrm{q} 24.1$, the chromosomal rearrangement involving MYBL1 in ACC064 was considered complex.

In the remaining eight cases, full-length $M Y B$ (ACC024, ACC098, ACC102, ACC110, ACC120, and ACC122) and truncated MYBL1 (ACC003 and ACC051) were highly expressed. In a case with the full-length $M Y B$ transcript (ACC098), an MPZL1 (1q24.2) exon 4-NFIB exon 4 fusion transcript was detected (Table 2; Supplementary Figure 6). In confirmatory RT-PCR, a transcript with an insertion of 69bases from 6q25.3 between MPZL1 exon 4 and NFIB exon 4 was also identified (Supplementary Figure 4C; Supplementary Figure 6).

Taken together, the following results were obtained in the analyses of $M Y B$ and $M Y B L 1$ transcripts in the 27 cases. Either $M Y B$ or MYBL1 transcripts were expressed in all cases in a mutually exclusive manner, regardless of its form (full-length, truncation, or fusion). In the fusion forms, both in-frame and out-of-frame fusions were identified. In all $M Y B$ and MYBL1 transcripts, the DNA-binding domain and trans-activation domain were retained (Table 2; Fig. 1).

\section{FISH for MYB and MYBL1 loci in the 27 cases}

We subsequently performed FISH assays using formalinfixed paraffin-embedded specimens to determine whether 

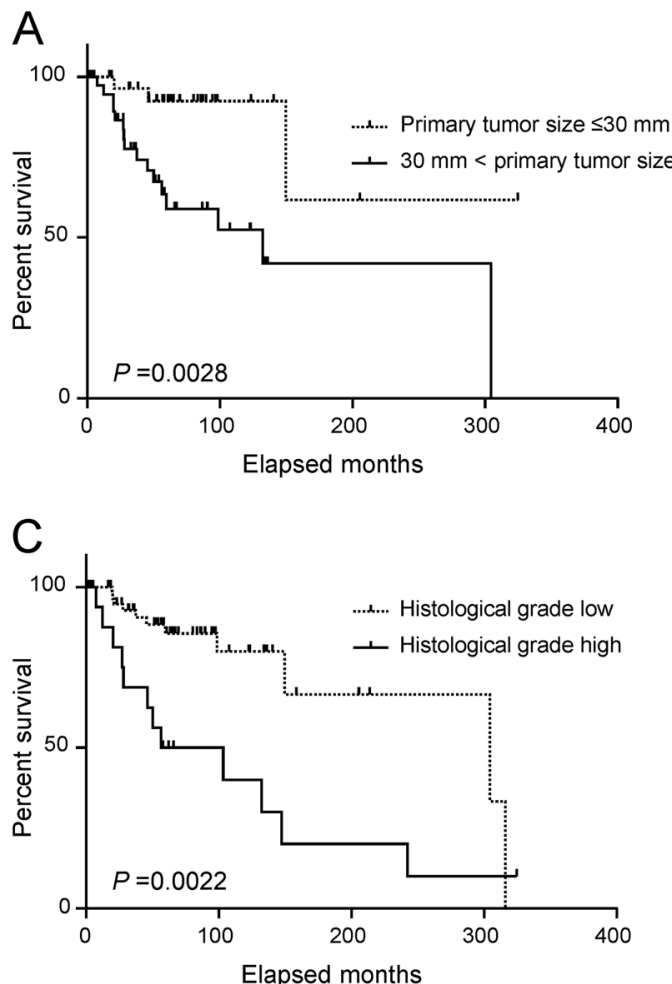

Fig. 4 Overall survival curves in the $78 M Y B / M Y B L 1$ locus rearrangement-positive cases whose primary sites were the head and neck (including orbital region). Cases with clinicopathological factors:

genomic rearrangements occurred around $M Y B$ and $M Y B L 1$ in these 27 cases. In adenoid cystic carcinoma, recent studies reported the fusion transcripts of $N F I B$ and genes around MYB (6q23.3), such as NKAIN2 (6q22.31) and AIG1 (6q24.2) [13]. These data prompted us to design probes that covered the $\sim 20 \mathrm{Mb}$ region around MYB (Supplementary Figure 1A), which was much broader than those covered by previously reported and commercially available FISH probes (Supplementary Figure 1B) [11, 16-20]. Among the 27 cases, 17 and 8 were positive in MYB and MYBL1 split FISH assays, respectively (Table 2; Supplementary Figure 7). One of the remaining two cases (ACC008) was negative for $M Y B$ split signals, but was positive in the $M Y B$ $N F I B$ fusion FISH assay. The other case (ACC037) was negative for $M Y B L 1$ split signals, but was positive in the MYBL1-NFIB fusion FISH assay.

Collectively, genomic rearrangements around the corresponding highly-expressed gene were observed in all 27 examined adenoid cystic carcinoma cases (18 cases for $M Y B$ and 9 cases for $M Y B L 1$ ), and the NFIB locus (9p2322.3) was involved in 16 of $18 M Y B$ and 7 of 9 MYBL1 locus rearrangements (Table 2). In addition, it was shown that $M Y B$ and $M Y B L 1$ were the target genes for overexpression in $M Y B$ and $M Y B L 1$ locus rearrangements, but not the other adjacent genes around MYB (ALDH8Al, HBSIL, AHII, and LINC00271) and MYBLI (TRIM55,

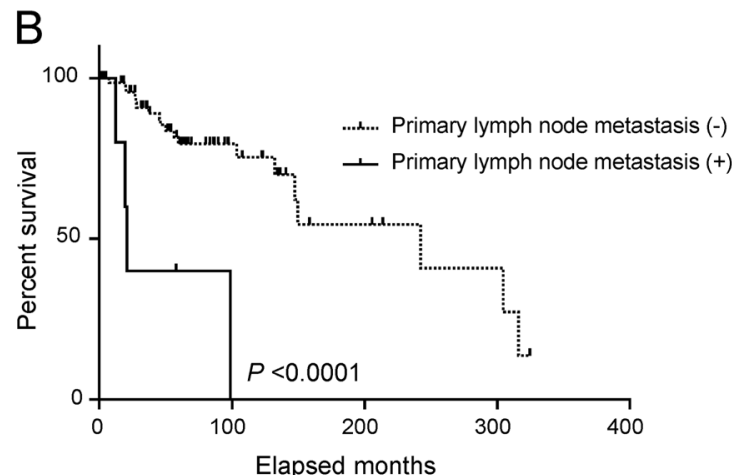

a $>30 \mathrm{~mm}$ primary tumor size $(N=38)(P=0.0028)$, b primary lymph node metastasis $(N=5)(P<0.0001)$, and $\mathbf{c}$ high histological grade $(N=18)(P=0.0022)$

ADHFE1, C8orf46, and C8orf44-SGK3), respectively (Supplementary Table 5; Supplementary Figure 8).

\section{Validation of $M Y B$ and $M Y B L 1$ rearrangements using 73 additional cases}

On the basis of the fact that all of the examined 27 adenoid cystic carcinoma cases harbored genomic rearrangements in the MYB or MYBLI locus, we further performed FISH analyses using formalin-fixed paraffin-embedded specimens of 73 additional adenoid cystic carcinoma cases. MYB split signals and $M Y B L 1$ split signals were identified in 52 and 15 cases, respectively. In ACC062 and ACC101, MYB$N F I B$ fusion signals were positive, although $M Y B$ split signals were negative as in ACC008. In ACC055, MYBL1$N F I B$ fusion signals were positive, although $M Y B L 1$ split signals were negative as in ACC037 (Fig. 3).

Among the 100 cases examined, 97 showed either of the following signal patterns: both $M Y B$ split and $M Y B-N F I B$ fusion signals in 57 , only $M Y B$ split signals in 12 , only $M Y B-N F I B$ fusion signals in 3, both MYBL1 split and MYBL1-NFIB fusion signals in 18, only MYBL1 split signals in 5, and only MYBL1-NFIB fusion signals in 2. The remaining three cases (ACC020, ACC114, and ACC119) were negative for any of these split/fusion FISH signals (Fig. 3). 


\section{Further analyses of genomic rearrangements in cases with atypical FISH patterns}

Thirteen cases with atypical FISH patterns were further analyzed by capture RNA sequencing (Table 2). In the five cases negative for $M Y B$ split with the conventional probe set but positive with the wide-range set (ACC024, ACC056, ACC093, ACC102, and ACC112), full-length $M Y B$ expression was detected except for ACC093 and ACC112, which were not evaluable due to the low quality of RNA.

$M Y B$ split was negative but MYB-NFIB fusion was positive in three cases (ACC008, ACC062, and ACC101). In ACC008, the discrepant FISH results support our abovementioned estimation that a $\sim 900 \mathrm{~kb}$ fragment of chromosome 9p23-p22.3 (3.6 kb downstream from NFIB 3'UTR to $M P D Z$ exon 24) was probably inserted in $M Y B$ intron 14 (Fig. 2a), as the insertion size is far below the resolution limit of the split FISH assay on formalin-fixed paraffinembedded slices. In ACC062, an MYB exon 8-NFIB exon 12 fusion transcript was identified, indicating that a small fragment containing $N F I B$ exon 12 and after was inserted in MYB intron 8 through a similar mechanism as in ACC008. In ACC101, full-length $M Y B$ was highly expressed and an NFIB exon 4-LINC01235 exon 2 fusion transcript was identified. LINC01235 is located $650 \mathrm{~kb}$ downstream from $N F I B$, and therefore the region from $N F I B$ intron 4 to LINC01235 intron 1 may be juxtaposed to MYB (Fig. 2b).

MYBL1 split was negative while MYBL1-NFIB fusion was positive in two cases (ACC037 and ACC055). A MYBL1 exon 14-NFIB exon 9 fusion transcript was expressed in ACC037. Therefore, as in the three cases (ACC008, ACC062, and ACC101), a small fragment containing $N F I B$ exon 9 and after was likely inserted into MYBL1 intron 14. Capture RNA sequencing was not successful in ACC055 for unknown reasons.

In the three cases negative in all FISH analyses (ACC020, ACC114, and ACC119), the expression of fulllength $M Y B$ mRNA and MYB protein was detected (Supplementary Figure 9). Any causative genomic rearrangements for the expression of $M Y B$ were not observed in the present FISH assays or capture RNA sequencing. Interestingly, all three FISH-negative cases were of external ear canal origin, and the remaining external ear canal case was also atypical in FISH (ACC024), which was negative for $M Y B$ split with the conventional probe set but positive with the wide-range set.

\section{Secondary MYBL1 locus rearrangement as a passenger event}

In the 97 cases showing rearrangements, those in the $M Y B$ and $M Y B L 1$ loci were fundamentally mutually exclusive (Fig. 3), except for one case (ACC018). The sample from the primary lesion (lacrimal gland) was positive in $M Y B$ split FISH and negative in MYB-NFIB fusion FISH and $M Y B L 1$ split FISH. However, MYBL1 split signals were also detected in samples from relapsed lesions. We examined all four lesions (primary and relapsed at 2, 4, and 6 years after) by capture RNA sequencing with TruSight RNA PanCancer Panel using RNA extracted from formalin-fixed paraffin-embedded tissues. Full-length $M Y B$ mRNA expression was constantly detected, while MYBL1 expression remained very low (Supplementary Figure 10). Therefore, the MYBL1 locus rearrangement in this MYBrearranged case was likely a secondary and passenger event. A fusion transcript of $R A D 51 B$ exon 7 (14q24.1) fused to $A H I 1$ exon 23 (114 kb downstream from MYB) was detected, which was confirmed by RT-PCR (Supplementary Figure 4D), supporting the FISH results (positive for $M Y B$ split and negative for MYB-NFIB fusion).

\section{Clinicopathological analysis of 78 head and neck cases with confirmed MYB/MYBL1 locus rearrangement}

Prognostic analyses were performed on 78 head and neck cases (including four cases in the ocular regions) with confirmed $M Y B$ or MYBL1 rearrangement. Three variables, primary tumor size $(30 \mathrm{~mm}<, P=0.0028)$, primary lymph node metastasis (positive, $P<0.0001$ ), and histological grade (high, $P=0.0022$ ) were significantly associated with poorer overall survival in univariate analysis (Supplementary Table 6; Fig. 4). In multivariate analysis of these three variables, primary tumor size $(P=0.003)$, primary lymph node metastasis $(P=0.002)$, and histological grade $(P<$ 0.001 ) were independent prognostic factors.

Any clinical features tested including overall survival did not significantly differ between the cases with $M Y B$ and MYBL1 alterations in the present cohort. However, histological grade was generally higher in cases with $M Y B$ alterations $(P=0.10$, Fisher's exact test; $P=0.023$, Mann-Whitney $U$ test) (Supplementary Table 7). Given that histological grade was found to be an independent prognostic factor, a large-scale study with detailed examination of $M Y B / M Y B L 1$ alterations would reveal the prognostic significance of the alteration.

\section{Discussion}

In the present study, we found that 97 of the 100 adenoid cystic carcinoma cases were associated with genomic rearrangement either in the MYB locus (73 cases) or in the MYBL1 locus (24 cases). In addition, all 27 cases examined expressed MYB $(18 / 27,67 \%)$ or MYBL1 $(9 / 27,33 \%)$ transcripts in a mutually exclusive manner. There were various 
forms of the transcripts including full-length, truncated, and fusion forms. In fusion forms, both in-frame and out-offrame fusion transcripts were detected. In all transcripts, the DNA-binding domain and trans-activation domain were retained. Taken together, these observations suggest that the fundamental alteration in the development of adenoid cystic carcinoma is dysregulated overexpression of the two domains in MYB or MYBL1 led by translocation of superenhancers (mostly those of $N F I B$ ) in the proximity of $M Y B$ or $M Y B L 1$.

Given its high frequency, MYB/MYBLl locus rearrangement can be a sensitive diagnostic marker of adenoid cystic carcinoma. However, the diversity of the rearrangements was also revealed and thus the method of detection should be designed judiciously. Although the frequency of $M Y B-N F I B$ fusion transcript in adenoid cystic carcinoma in the initial report was $100 \%(11 / 11)$ [8], the frequency obtained by RT-PCR was relatively low considering subsequent reports $(51.1 \%, 162 / 317)$ [8-10, 12, 16, 18, 19, 2731]. Aside from technical issues (primer design, formalinfixed paraffin-embedded vs. frozen materials, etc.), this was explained by the presence of other types of $M Y B$ expression including full-length $M Y B$, truncated $M Y B$, and $M Y B$ fusion transcript with other partner genes (PDCD1LG2 at 9p24.1, EFR3A at 8q24.22, TGFBR3 at 1p22.1, and $R A D 51 B$ at $14 \mathrm{q} 24.1)$ [28, 32]. In addition, the MYBL1$N F I B$ fusion was reported in 2015. The reported frequency of the fusion varies depending on methodology used $(10.9 \%$, 16/147, transcript-based; 11.9\%, 16/135, $M Y B L 1-N F I B$ fusion FISH), and other types of $M Y B L 1$ expression including truncated $M Y B L 1$ and $M Y B L 1$ fusion with other partner genes have been reported [12-14, 33]. Although $M Y B / M Y B L 1$ alterations are highly characteristic to adenoid cystic carcinoma, this diversity in $M Y B / M Y B L 1$ transcripts dampens the efficacy of RT-PCR-based approaches for confirmatory diagnosis of adenoid cystic carcinoma.

In the present study, we detected 10 cases (10\%) whose $M Y B / M Y B L 1$ locus rearrangements could not be detected using the conventionally designed split FISH probe set (Table 2). In five cases, genomic rearrangements in the $M Y B$ locus turned out to be visible with the wide-range probe set. The probe set covered the $\sim 20 \mathrm{Mb}$ region around $M Y B$ (Supplementary Figure 1A), which was extremely broad compared with those covered by previously reported and commercially available FISH probes (Supplementary Figure 1B) [11, 16-20]. Thus, the breakpoints in the $M Y B$ locus are far distant from $M Y B(\sim 10 \mathrm{Mb})$ in these cases. In the other five cases, a small insertion $(<1 \mathrm{Mb})$ that could not be recognized by split FISH assay was considered to occur within $M Y B$ (ACC008 and ACC062), close to $M Y B$ (ACC101), within MYBL1 (ACC037), and within or close to MYBL1 (ACC055). The super-enhancer elements in
NFIB, TGFBR3, or RAD51B loci translocated to the proximity of $M Y B$ (regardless of whether it was upstream or downstream) caused overexpression of MYB protein, which binds the elements and sustains $M Y B$ expression in a positive feedback manner [32]. On the basis of previous results and those of the present study, $M Y B$ can be upregulated even if a genomic region containing the superenhancer elements is translocated "far distant" from $M Y B$ (but the elements should physically interact with $M Y B$ promoter region) and/or if the genomic region is too small to be observed by conventional karyotyping and FISH assays.

FISH assay is a powerful tool for detecting genomic rearrangements. Particularly, split FISH assay is suitable for screening for rearrangement of genes of interest, as it is available regardless of the rearranged partners. For example, the split FISH assay for $A L K$ has been widely used as a companion diagnostic for $A L K$ inhibitors in ALK-positive lung cancers, in which $A L K$ is rearranged with various potential fusion partner genes including EMLA [34], KIF5B [35], KLC1 [21], and others. However, the presence of above-mentioned atypical patterns of $M Y B / M Y B L 1$ locus rearrangement in adenoid cystic carcinoma may limit FISH as a confirmatory diagnostic assay. Using conventionally designed split FISH probe sets, the frequency of $M Y B$ / MYBL1 locus rearrangement in our cohort was $87 \%(87 /$ 100 ), which is comparable to that in a study in which both $M Y B$ and MYBLl were examined by FISH $(88 \%, 29 / 33)$ [33]. Therefore, to maintain the sensitivity of FISH for detecting $M Y B / M Y B L 1$ locus rearrangement in adenoid cystic carcinoma, combined analysis using the split FISH assay with a wide-range probe set and fusion FISH assay using probes for known fusion partners (at least NFIB) is necessary. For example, one of the practical ways is following; 3-color FISH using a mixture of a conventionally designed $M Y B$ probe set (e.g., set A in Supplementary Table 2) and NFIB (e.g., set C) is performed in a case. If the result is negative, a mixture of a $M Y B L 1$ probe set (e.g., set B) and $N F I B$ (e.g., set C) is performed. If the result is negative, FISH using wide-range $M Y B$ probe set is performed. The first two or all three FISH tests can be done at the same time.

Among the 14 excluded cases without a consensus diagnosis of adenoid cystic carcinoma, 11 were evaluable and negative for $M Y B / M Y B L 1$ rearrangement (data not shown). Capture RNA sequencing was performed in 4 cases. MYBL1 expression was not detected in the 4 cases, but the expression of full-length $M Y B$ transcript was detected in 3 cases. This means that the expression-based assays that cannot distinguish MYB forms (full length, fusion, and truncation), such as anti-MYB immunohistochemistry, are less useful in differentiating adenoid cystic carcinoma from its mimics in diagnostic settings. 
Acknowledgements We thank Mr. Motoyoshi Iwakoshi, Ms. Keiko Shiozawa, and Ms. Tomoyo Kakita for their technical assistance and Ms. Sayuri Sengoku for her management assistance in the research project. We also thank Dr. Hiroyuki Yonekawa, Dr. Hirofumi Fukushima, Dr. Toru Sasaki, Dr. Wataru Shinbashi, Dr. Kazutoshi Toda, Dr. Aya Ebina, and Dr. Hideki Tsuji for providing clinical specimens and Dr. Futoshi Akiyama and Dr. Yutaka Takazawa for giving advice on histological review. Computations were partially performed on the NIG supercomputer at ROIS National Institute of Genetics. This study was supported in part by the Japan Society for the Promotion of Science KAKENHI grant number 16K21591 (to Yuki Togashi).

\section{Compliance with ethical standards}

Conflict of interest The authors declare that they have no conflict of interest.

\section{References}

1. Lloyd S, Yu JB, Wilson LD, et al. Determinants and patterns of survival in adenoid cystic carcinoma of the head and neck, including an analysis of adjuvant radiation therapy. Am J Clin Oncol. 2011;34:76-81.

2. Kas K, Voz ML, Roijer E, et al. Promoter swapping between the genes for a novel zinc finger protein and beta-catenin in pleiomorphic adenomas with $\mathrm{t}(3 ; 8)(\mathrm{p} 21 ; \mathrm{q} 12)$ translocations. Nat Genet. 1997;15:170-4.

3. Geurts JM, Schoenmakers EF, Roijer E, et al. Expression of reciprocal hybrid transcripts of HMGIC and FHIT in a pleomorphic adenoma of the parotid gland. Cancer Res. 1997;57:13-7.

4. Nordkvist A, Gustafsson H, Juberg-Ode M, et al. Recurrent rearrangements of 11q14-22 in mucoepidermoid carcinoma. Cancer Genet Cytogenet. 1994;74:77-83.

5. Winnes M, Molne L, Suurkula M, et al. Frequent fusion of the CRTC1 and MAML2 genes in clear cell variants of cutaneous hidradenomas. Genes Chromosomes Cancer. 2007;46: 559-63.

6. Skalova A, Vanecek T, Sima R, et al. Mammary analogue secretory carcinoma of salivary glands, containing the ETV6NTRK3 fusion gene: a hitherto undescribed salivary gland tumor entity. Am J Surg Pathol. 2010;34:599-608.

7. Antonescu CR, Katabi N, Zhang L, et al. EWSR1-ATF1 fusion is a novel and consistent finding in hyalinizing clear-cell carcinoma of salivary gland. Genes Chromosomes Cancer. 2011;50:559-70.

8. Persson M, Andren Y, Mark J, et al. Recurrent fusion of MYB and NFIB transcription factor genes in carcinomas of the breast and head and neck. Proc Natl Acad Sci USA. 2009;106:18740-4.

9. Mitani Y, Li J, Rao PH, et al. Comprehensive analysis of the MYB-NFIB gene fusion in salivary adenoid cystic carcinoma: Incidence, variability, and clinicopathologic significance. Clin Cancer Res. 2010;16:4722-31.

10. Brill LB 2nd, Kanner WA, Fehr A, et al. Analysis of MYB expression and MYB-NFIB gene fusions in adenoid cystic carcinoma and other salivary neoplasms. Mod Pathol. 2011;24:1169-76.

11. West RB, Kong C, Clarke N, et al. MYB expression and translocation in adenoid cystic carcinomas and other salivary gland tumors with clinicopathologic correlation. Am J Surg Pathol. 2011;35:92-9.
12. Brayer KJ, Frerich CA, Kang H, et al. Recurrent fusions in MYB and MYBL1 define a common, transcription factor-driven oncogenic pathway in salivary gland adenoid cystic carcinoma. Cancer Discov. 2016;6:176-87.

13. Mitani Y, Liu B, Rao PH, et al. Novel MYBL1 gene rearrangements with recurrent MYBL1-NFIB Fusions in salivary adenoid cystic carcinomas lacking $\mathrm{t}(6 ; 9)$ translocations. Clin Cancer Res. 2016;22:725-33.

14. Rettig EM, Talbot CC Jr., Sausen M, et al. Whole-genome sequencing of salivary gland adenoid cystic carcinoma. Cancer Prev Res. 2016;9:265-74.

15. Wysocki PT, Izumchenko E, Meir J, et al. Adenoid cystic carcinoma: emerging role of translocations and gene fusions. Oncotarget. 2016;7:66239-54.

16. von Holstein SL, Fehr A, Persson M, et al. Adenoid cystic carcinoma of the lacrimal gland: MYB gene activation, genomic imbalances, and clinical characteristics. Ophthalmology. 2013;120:2130-8.

17. Zhang J, Wu G, Miller CP, et al. Whole-genome sequencing identifies genetic alterations in pediatric low-grade gliomas. Nat Genet. 2013;45:602-12.

18. Martelotto LG, De Filippo MR, Ng CK, et al. Genomic landscape of adenoid cystic carcinoma of the breast. J Pathol. 2015;237:179-89.

19. North JP, McCalmont TH, Fehr A, et al. Detection of MYB alterations and other immunohistochemical markers in primary cutaneous adenoid cystic carcinoma. Am J Surg Pathol. 2015;39:1347-56.

20. Rettig EM, Tan M, Ling S, et al. MYB rearrangement and clinicopathologic characteristics in head and neck adenoid cystic carcinoma. Laryngoscope. 2015;125:E292-9.

21. Togashi Y, Soda M, Sakata S, et al. KLC1-ALK: a novel fusion in lung cancer identified using a formalin-fixed paraffin-embedded tissue only. PLoS ONE. 2012;7:e31323.

22. Schmieder R, Edwards R. Quality control and preprocessing of metagenomic datasets. Bioinformatics. 2011;27:863-4.

23. Dobin A, Davis CA, Schlesinger F, et al. STAR: ultrafast universal RNA-seq aligner. Bioinformatics. 2013;29:15-21.

24. Daniel Nicorici MS, Henrik Edgren, Sara Kangaspeska, et al. FusionCatcher-a tool for finding somatic fusion genes in pairedend RNA-sequencing data. bioRxiv. 2014.

25. RC Team. R: A Language and Environment for Statistical Computing (R Foundation for Statistical Computing, Vienna, Austria, 2016).

26. Anders S, Pyl PT, Huber W. HTSeq-a Python framework to work with high-throughput sequencing data. Bioinformatics. 2015;31:166-9.

27. Fehr A, Kovacs A, Loning T, et al. The MYB-NFIB gene fusion - a novel genetic link between adenoid cystic carcinoma and dermal cylindroma. J Pathol. 2011;224:322-7.

28. Mitani Y, Rao PH, Futreal PA, et al. Novel chromosomal rearrangements and break points at the $\mathrm{t}(6 ; 9)$ in salivary adenoid cystic carcinoma: association with MYB-NFIB chimeric fusion, MYB expression, and clinical outcome. Clin Cancer Res. 2011;17:7003-14.

29. Persson M, Andren Y, Moskaluk CA, et al. Clinically significant copy number alterations and complex rearrangements of MYB and NFIB in head and neck adenoid cystic carcinoma. Genes Chromosomes Cancer. 2012;51:805-17.

30. Wetterskog D, Lopez-Garcia MA, Lambros MB, et al. Adenoid cystic carcinomas constitute a genomically distinct subgroup of triple-negative and basal-like breast cancers. J Pathol. 2012;226:84-96.

31. D'Alfonso TM, Mosquera JM, MacDonald TY, et al. MYB-NFIB gene fusion in adenoid cystic carcinoma of the breast with special 
focus paid to the solid variant with basaloid features. Hum Pathol. 2014;45:2270-80.

32. Drier Y, Cotton MJ, Williamson KE, et al. An oncogenic MYB feedback loop drives alternate cell fates in adenoid cystic carcinoma. Nat Genet. 2016;48:265-72.

33. Fujii K, Murase T, Beppu S, et al. MYB, MYBL1, MYBL2 and NFIB gene alterations and MYC overexpression in salivary gland adenoid cystic carcinoma. Histopathology. 2017;71:823-34.
34. Soda M, Choi YL, Enomoto M, et al. Identification of the transforming EML4-ALK fusion gene in non-small-cell lung cancer. Nature. 2007;448:561-6.

35. Takeuchi K, Choi YL, Togashi Y, et al. KIF5B-ALK, a novel fusion oncokinase identified by an immunohistochemistry-based diagnostic system for ALK-positive lung cancer. Clin Cancer Res. 2009;15:3143-9. 